\title{
Can Computed Tomography Colonography Replace Optical Colonoscopy in Detecting Colorectal Lesions?: State of the Art
}

\author{
Alessia Chini', Michele Manigrasso ${ }^{2}$, Grazia Cantore', Rosa Maione', Marco Milone', Francesco Maione ${ }^{1}$, and Giovanni Domenico \\ De Palma ${ }^{1}$ \\ ${ }^{1}$ Department of Clinical Medicine and Surgery, University of Naples "Federico II", Naples, ${ }^{2}$ Department of Advanced Biomedical \\ Sciences, University of Naples "Federico II", Naples, Italy
}

Colorectal cancer is an important cause of morbidity and mortality worldwide. Optical colonoscopy (OC) is widely accepted as the reference standard for the screening of colorectal polyps and cancers, and computed tomography colonography (CTC) is a valid alternative to OC. The purpose of this review was to assess the diagnostic accuracy of OC and CTC for colorectal lesions. A literature search was performed in PubMed, Embase, and Cochrane Library, and 18 articles were included. CTC has emerged in recent years as a potential screening examination with high accuracy for the detection of colorectal lesions. However, the clinical application of CTC as a screening technique is limited because it is highly dependent on the size of the lesions and has poor performance in detecting individual lesions $<5 \mathrm{~mm}$ or flat lesions, which, although rarely, can have a malignant potential. Clin Endosc 2022;55:183-190

Key Words: Colonoscopy; Colorectal cancer; Colorectal polyps; Computed tomography colonography

\section{INTRODUCTION}

Colorectal cancer (CRC) is a major cause of cancer-related morbidity and mortality worldwide, being the fourth most common cause of cancer death and the third most common cancer globally. ${ }^{1}$ The major risk factors for CRC are obesity, alcohol consumption, cigarette smoking, and a sedentary lifestyle, and this cancer is more prevalent in Western countries. ${ }^{2}$ However, CRC is also increasingly affecting Asian populations. The overall prevalence and incidence of CRC are reported to be lower in Asian than in Western populations, although Asian countries, especially China, Japan, and Korea, have the

Received: October 11, 2021 Revised: November 30, 2021

Accepted: December 1, 2021

Correspondence: Alessia Chini

Department of Clinical Medicine and Surgery, "Federico II" University of Naples, Via Sergio Pansini, 5, Naples 80131, Italy

Tel: +39-329-212-2828, Fax: +39-081-746-2716, E-mail: alessiachini@hotmail.it ORCID: https://orcid.org/0000-0002-1851-1007

(cc) This is an Open Access article distributed under the terms of the Creative Commons Attribution Non-Commercial License (http://creativecommons.org/ licenses/by-nc/3.0) which permits unrestricted non-commercial use, distribution, and reproduction in any medium, provided the original work is properly cited. highest number of prevalent cases. ${ }^{3}$ It is widely accepted that most CRCs develop from preexisting adenomatous colon polyps through the adenoma-to-carcinoma sequence, a multistep process that takes many years to complete, during which a sequential accumulation of genetic mutations occur. The transformation from normal epithelium to hyperproliferative mucosa can eventually result in the formation of carcinoma. ${ }^{4,5}$ For this reason, colorectal adenomatous polyps are the optimal target lesions for strategies to prevent CRC. ${ }^{6}$ The currently available screening tests for CRC are stool-based tests (e.g., stool DNA tests, guaiac-based fecal occult blood test [FOBT], and immunochemical FOBT) and structural examinations (e.g., flexible sigmoidoscopy, optical colonoscopy [OC], double-contrast barium enema, and computed tomography [CT] colonography [CTC], also known as virtual colonoscopy). The colon capsule endoscopy is another technique that allows the evaluation of the whole colon; however, it is not an accepted method for CRC screening. ${ }^{7}$ Colonoscopy is widely accepted as the gold standard for the detection of CRC and has the advantage of being both diagnostic and therapeutic because biopsy and resection of polyps or early cancer can be performed during the same procedure. Although OC has a 
high accuracy in detecting colorectal lesions, it is an invasive technique with a risk of bowel perforation and bleeding. CTC is a good alternative to colonoscopy for CRC screening, as it is a safe and minimally invasive technique that allows the evaluation of the whole colon, including in cases in which OC is not possible to perform; ${ }^{8}$ however, some limitations of CTC remain a problem. For example, the total amount of ionizing radiation delivered to patients is a concern, although technological improvements, including the implementation of image reconstruction algorithms and reduction in tube current, have contributed to reducing the associated radiation exposure. ${ }^{9}$ Several studies have shown that CTC is comparable to OC in terms of accuracy in detecting polyps and CRC; however, it also presents advantages over OC, such as the certainty of a complete examination and the identification of additional extraluminal pathology. The purpose of this review was to compare OC and CTC in the detection of colorectal lesions, both in asymptomatic average-risk patients and symptomatic highrisk patients.

\section{MATERIALS AND METHODS}

To identify all studies that compared OC and CTC in terms of accuracy in detecting colorectal lesions, a comprehensive literature search was conducted in electronic databases (PubMed/MEDLINE, Google Scholar, Embase, and Cochrane Library), according to the PRISMA (Preferred Reporting Items for Systematic Reviews and Meta-analyses) guidelines, ${ }^{10}$ without any restrictions with respect to the publication year. Lists of synonyms for CTC and colonoscopy were produced and combined using the Boolean operator "OR", and the search results were combined using the Boolean operator "AND". All types of observational studies that compared CTC and OC for the screening and detection of colorectal polyps, CRC, and advanced neoplasia, both in asymptomatic average-risk patients and symptomatic high-risk patients, restricted to articles written in English, were included in the review. Articles not reporting a comparison between the two techniques, meta-analysis articles, case reports, letters to the editor, and commentaries were excluded. With respect to the details of the two procedures, OC is usually performed by introducing a flexible endoscope through the anus after adequate lubrication and digital inspection of the perianal and rectal regions. Correct endoscope manipulation by the endoscopist in OC allows the exploration, in a retrograde sense, of all segments of the large intestine until the cecum is reached. ${ }^{11}$ Meanwhile, CTC uses volumetric CT data produced by a helical CT scan- ner to generate three-dimensional images displayed in a cine loop, combined with advanced imaging software to generate two-dimensional and three-dimensional images of the colon and rectum. The patient is placed in the left lateral position, and the colon is gently insufflated with air by inserting a Foley catheter in the rectum, according to patient tolerance. Scanning is performed in the prone and supine positions to obtain video sequences similar to those in OC, in two-dimensional and three-dimensional reconstructions. Bowel preparation is required for both CTC and OC. Although good bowel preparation is essential in both procedures, OC has a disadvantage in that the presence of excessive residual fluid hinders the advancement of the colonoscope, whereas CTC does not have this problem. Furthermore, intravenous contrast enhancement in CTC could differentiate residual fluid from colorectal diseases and CRC from inflammatory pathologies, and several studies have shown that intravenous contrast improves the detection of colonic lesions with CTC. ${ }^{12}$

\section{RESULTS}

The search yielded 1,085 articles, of which 75 were excluded for being duplicates. Two authors independently reviewed the remaining articles by first reading the titles and abstracts. Articles that were not pertinent (not comparing OC and CTC in the detection of colorectal lesions) or not written in English were further excluded. A total of 48 potentially relevant papers were identified, which were retrieved as full-text articles. The reference lists of the final selection of articles were manually checked to identify other relevant papers. Any disagreement was resolved by consensus with a third author (Fig. 1). A total of 18 articles were included in this review. Table 1 reports the characteristics of the included studies: publication year, type of study, number and mean age of the patients included in each study, and whether the patients underwent the procedures in a screening setting. The results are expressed in two ways: individual polyp detection (per-polyp) and patient detection (per-patient). Table 2 reports the per-polyp sensitivity, classified according to the size of each polyp detected with CTC and OC, from the studies included in this review. In particular, information on sensitivity was collected for both CTC and OC from each study, using the following thresholds: $\leq 5,6-9 \mathrm{~mm}$, and $\geq 10 \mathrm{~mm}$. The overall sensitivity is also reported. Table 3 shows the overall per-patient sensitivity, specificity, positive predictive value, and negative predictive value of CTC and OC for the detection of colorectal lesions. 


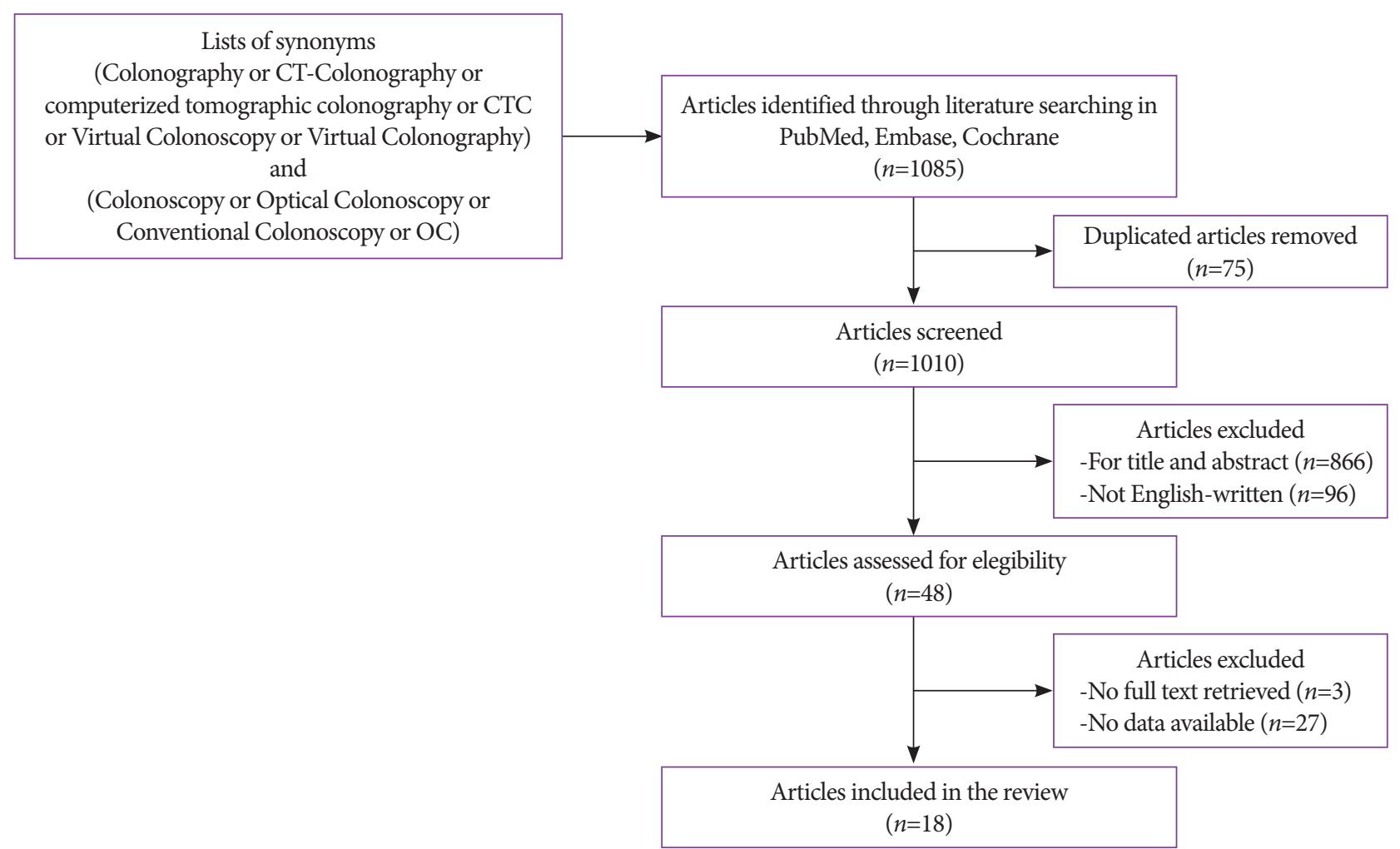

Fig. 1. Study selection flowchart according to preferred reporting items for systematic reviews and meta-analyses (PRISMA). CT, computed tomography; CTC, computed tomography colonography; $\mathrm{OC}$, optical colonoscopy.

Table 1. Characteristics of the Included Studies

\begin{tabular}{llccc}
\hline Study & \multicolumn{1}{c}{ Type of study } & No. of patients & Mean age, years (range) & Screening setting \\
\hline Munikrishnan et al. $(2003)^{13}$ & Single-center, prospective & 80 & $68(29-83)$ & No \\
\hline Singh et al. $(2015)^{14}$ & Single-center, prospective & 50 & - & No \\
\hline Laghi et al. $(2002)^{15}$ & Single-center, prospective & 66 & $61(30-84)$ & No \\
\hline Macari et al. $(2002)^{16}$ & Single-center, prospective & 105 & $58(49-79)$ & No \\
\hline Graser et al. $(2009)^{17}$ & Single-center, prospective & 311 & $60.5(50-81)$ & Yes \\
\hline Iannaccone et al. $(2003)^{18}$ & Single-center, prospective & 158 & $64(50-80)$ & No \\
\hline Fenlon et al. $(1999)^{19}$ & Single-center, prospective & 100 & $62(50-77)$ & No \\
\hline Yee et al. $(2001)^{20}$ & Single-center, prospective & 300 & $62.6(25-90)$ & No \\
\hline Pickhardt et al. $(2003)^{21}$ & Multicenter, prospective & 1253 & $57.8(40-79)$ & Yes \\
\hline Pineau et al. $(2003)^{22}$ & Single-center, prospective & 206 & $59.3(38-83)$ & No \\
\hline Ginnerup Pedersen et al. $(2003)^{23}$ & Single-center, prospective & 148 & $60(25-86)$ & No \\
\hline Devir et al. $(2016)^{24}$ & Single-center, prospective & 31 & $45(38-74)$ & Yes \\
\hline Gluecker et al. $(2002)^{25}$ & Single-center, prospective & 51 & $60(50-75)$ & No \\
\hline Hoppe et al. $(2004)^{26}$ & Single-center, prospective & 100 & $66(20-91)$ & No \\
\hline Chaparro Sánchez et al. $(2007)^{27}$ & Single-center, prospective & 50 & $62(25-83)$ & No \\
\hline Johnson et al. $(2003)^{28}$ & Single-center, prospective & 703 & $64(50-84)$ & No \\
\hline Spinzi et al. $(2001)^{29}$ & Single-center, prospective & 99 & 615 & No \\
\hline Cotton et al. $(2004)^{30}$ & Multicenter, prospective & 61 & No \\
\hline
\end{tabular}


Table 2. Per-polyp (\%) Sensitivity of Computed Tomography Colonography and Optical Colonoscopy

\begin{tabular}{|c|c|c|c|c|c|c|c|c|}
\hline \multirow{2}{*}{ Study } & \multicolumn{4}{|c|}{ Computed tomography colonography } & \multicolumn{4}{|c|}{ Optical colonoscopy } \\
\hline & Overall & $\leq 5 \mathrm{~mm}$ & $6-9 \mathrm{~mm}$ & $\geq 10 \mathrm{~mm}$ & Overall & $\leq 5 \mathrm{~mm}$ & 6-9 mm & $\geq 10 \mathrm{~mm}$ \\
\hline Munikrishnan et al. $(2003)^{13}$ & 76 & 53 & 83 & 100 & - & - & - & - \\
\hline Singh et al. $(2015)^{14}$ & 97.5 & - & - & - & 92.68 & - & - & - \\
\hline Laghi et al. $(2002)^{15}$ & 57.6 & 24 & 84.6 & 92.8 & - & - & - & - \\
\hline Macari et al. $(2002)^{16}$ & 21 & 12 & 53 & 100 & - & - & - & - \\
\hline Graser et al. $(2009)^{17}$ & 70.1 & 59.2 & 90.2 & 93.9 & 95.9 & 94.6 & 92.7 & 100 \\
\hline Iannaccone et al. $(2003)^{18}$ & 70 & 51 & 83 & 100 & - & - & - & - \\
\hline Fenlon et al. $(1999)^{19}$ & 71 & 55 & 82 & 91 & - & - & - & - \\
\hline Yee et al. $(2001)^{20}$ & 69.7 & 59.1 & 80.1 & 90 & - & - & - & - \\
\hline Pickhardt et al. $(2003)^{21}$ & 91.5 & 85.7 & 92.6 & 92.2 & 88.1 & 90 & 89.5 & 88.2 \\
\hline Pineau et al. $(2003)^{22}$ & 63.6 & 29.4 & 75 & 87.5 & - & - & - & - \\
\hline Ginnerup Pedersen et al. $(2003)^{23}$ & 81 & - & 71 & 83 & 87 & - & 100 & 70 \\
\hline Devir et al. $(2016)^{24}$ & 83.3 & 87.5 & 75 & 91.7 & - & - & - & - \\
\hline Gluecker et al. $(2002)^{25}$ & 22 & 4 & 33 & 82 & - & - & - & - \\
\hline Hoppe et al. $(2004)^{26}$ & 43 & 25 & 50 & 71 & 94 & - & - & - \\
\hline Chaparro Sánchez et al. $(2007)^{27}$ & - & 15 & 75 & 75 & - & - & - & - \\
\hline Johnson et al. $(2003)^{28}$ & 57.8 & - & 54 & 63 & - & - & - & - \\
\hline Spinzi et al. $(2001)^{29}$ & 58 & 56 & - & 62 & - & - & - & - \\
\hline Cotton et al. $(2004)^{30}$ & 39 & 7.6 & 22.7 & 52 & 98 & 97 & 99 & 96 \\
\hline
\end{tabular}

Table 3. Per-patient (\%) Sensitivity, Specificity, Positive Predictive Value, and Negative Predictive Value of Computed Tomography Colonography and Optical Colonoscopy

\begin{tabular}{|c|c|c|c|c|c|c|c|c|}
\hline \multirow{2}{*}{ Study } & \multicolumn{4}{|c|}{ Computed tomography colonography } & \multicolumn{4}{|c|}{ Optical colonoscopy } \\
\hline & Sensitivity & Specificity & PPV & NPV & Sensitivity & Specificity & PPV & NPV \\
\hline Munikrishnan et al. $(2003)^{13}$ & 97 & 98 & 96 & 98 & - & - & - & - \\
\hline Singh et al. $(2015)^{14}$ & 97.56 & 100 & 100 & 93.75 & 92.68 & 100 & 100 & 83.3 \\
\hline Laghi et al. $(2002)^{15}$ & 93.7 & 94.1 & - & - & - & - & - & - \\
\hline Macari et al. $(2002)^{16}$ & 32.5 & 97.7 & 93 & 85.3 & - & - & - & - \\
\hline Graser et al. $(2009)^{17}$ & 84.1 & 47.4 & 48.2 & 83.6 & 97.3 & 59.8 & 58.5 & 97.5 \\
\hline Iannaccone et al. $(2003)^{18}$ & 96 & 96.6 & 94.1 & 97.7 & - & - & - & - \\
\hline Fenlon et al. $(1999)^{19}$ & 82 & 84 & 82 & 84 & - & - & - & - \\
\hline Yee et al. $(2001)^{20}$ & 90.1 & 72 & 83.3 & 82.5 & - & - & - & - \\
\hline Pickhardt et al. $(2003)^{21}$ & 88.7 & 79.6 & - & - & 92.3 & - & - & - \\
\hline Pineau et al. $(2003)^{22}$ & 61.8 & 70.7 & 61.8 & 70.7 & - & - & - & - \\
\hline Ginnerup Pedersen et al. $(2003)^{23}$ & 91 & 97 & - & - & - & - & - & - \\
\hline Devir et al. $(2016)^{24}$ & 83 & 95 & 95 & 83 & - & - & - & - \\
\hline Gluecker et al. $(2002)^{25}$ & - & - & - & - & - & - & - & - \\
\hline Hoppe et al. $(2004)^{26}$ & 73 & 88 & 79 & 86 & - & - & - & - \\
\hline Chaparro Sánchez et al. $(2007)^{27}$ & 75 & 89 & - & - & - & - & - & - \\
\hline Johnson et al. $(2003)^{28}$ & - & - & - & - & - & - & - & - \\
\hline Spinzi et al. $(2001)^{29}$ & 57.8 & 92.6 & 86.7 & - & - & - & - & - \\
\hline Cotton et al. $(2004)^{30}$ & 39 & 90.5 & - & - & 99 & 100 & - & - \\
\hline
\end{tabular}

NPV, negative predictive value; PPV, positive predictive value. 


\section{DISCUSSION}

Colonoscopy and CTC are both highly accurate structural examinations that allow the inspection of the whole colon and are valid options for CRC screening. In particular, colonoscopy is considered the reference standard for the detection of colorectal polyps or CRC, despite being an invasive procedure associated with complications such as bleeding and perforation. Although OC is an accurate examination, it may miss $10-20 \%$ of colorectal polyps and $5 \%$ of colorectal tumors. ${ }^{31,32}$ Furthermore, $10-15 \%$ of colonoscopies (22-33\% in older patients) ${ }^{9}$ fail to be completed even if performed by experienced endoscopists, for several reasons (e.g., in cases of an abnormal colonic shape or length or in the presence of obstructing colonic tumors or stenosis); thus, in these cases, CTC is performed to evaluate the nonvisualized parts of the colon. ${ }^{33}$ Maggialetti et al. ${ }^{9}$ evaluated the role of CTC in patients who had previously undergone an incomplete OC and found that CTC allowed the complete evaluation of the nonvisualized parts of the colon in these patients, increasing the diagnostic yield of lesions in that part of the colon and allowing the assessment of extracolonic findings. In fact, one of the main advantages of CTC is that extracolonic findings can be simultaneously obtained, although they are prognostically relevant in only a few cases (2-5\%). CTC also offers the advantage of identifying the precise segmental location of colorectal tumors, which is not always possible with colonoscopy because of the difficulty in identifying anatomical landmarks. Correct preoperative localization of CRC is important in determining the adequate treatment during surgical planning, especially for laparoscopic procedures. Furthermore, CTC guarantees accurate tumor staging. ${ }^{14}$

Offermans et al. ${ }^{34}$ compared CTC and OC in terms of localization of colorectal lesions, using the surgical location to determine the segmental localization. They found that the error rate of CTC in segmental localization was lower than that of OC, especially when the neoplasms are located in the descending colon and cecum. CTC also has an established role in the preoperative evaluation of patients with occlusive CRC, allowing the visualization of the proximal colon. Several studies have reported that when an occlusive CRC is detected, 1.5-9\% of patients have a synchronous cancer and $27-55 \%$ have coexisting polyps. ${ }^{15}$ However, few studies have focused on the accuracy of CTC in diagnosing synchronous colonic lesions. In a recent study, Flor et al. ${ }^{35}$ showed that the per-patient sensitivity and specificity of CTC in detecting synchronous lesions $\geq 6$ mm were $93 \%$ and $98 \%$, respectively; those in detecting synchronous adenomatous lesions were $89 \%$ and $100 \%$, respectively; and those in detecting synchronous CRC were $94 \%$ and
$100 \%$, respectively. They concluded that CTC is a highly accurate procedure for detecting synchronous colonic lesions in patients with occlusive CRC. The detection of synchronous lesions is crucial for establishing the correct surgical management plan and avoiding subsequent reoperation. In addition, CTC has received considerable attention since its introduction in 1994, but has emerged only in recent years as a potential screening examination with high accuracy for the detection of colorectal lesions and a comparable diagnostic yield to colonoscopy for colorectal neoplasia but with a lower risk of complications. ${ }^{36}$ The sensitivity and specificity of CTC have improved during the past few years, owing to important advances in CT technology, especially in terms of imaging in multiple sections. In fact, single-section CTC has a low sensitivity for the detection of lesions $<10 \mathrm{~mm}$ and flat lesions and a high false-positive rate because of its inability to differentiate fecal material from polyps. The use of multidetector-row CT scanners that allow thinner section collimation has improved polyp detection through better bowel distension and fewer respiratory artifacts, due to faster data acquisition, and has decreased the false-positive rate. ${ }^{16}$ Another important concern is radiation exposure during CTC, which may lead to increased radiation-related risks of cancer and death. The radiation dose can be decreased by using low-dose protocols and new dose modulation techniques, by increasing the section collimation and pitch, and by decreasing the kilovolt or milliampere-second values. ${ }^{16,17}$ Macari et al. ${ }^{16}$ and Iannaccone et $\mathrm{al}^{18}$ reported that lower-dose multidetector-row CTC ensured substantial dose reduction while maintaining good sensitivity and specificity for the detection of colorectal lesions $>6 \mathrm{~mm}$ and excellent sensitivity and specificity for lesions $>10 \mathrm{~mm}$. One of the first studies to compare CTC and OC for the detection of colorectal lesions was conducted by Fenlon et al., ${ }^{19}$ who found that CTC and OC had a similar ability in detecting lesions $>6 \mathrm{~mm}$, although they admitted that the diagnostic accuracy in their study may have been overestimated because they included only high-risk patients. Yee et al. ${ }^{20}$ conducted the first large study on CTC as a screening test for colorectal lesions in asymptomatic average-risk patients and symptomatic high-risk patients. They found that the performance of CTC was comparable between the two groups. On the basis of their results, the authors affirmed that CTC is a valid screening examination in patients with a low risk of CRC. Meanwhile, Van Gelder et al. ${ }^{37}$ reported that CTC and OC had similar ability in identifying colorectal lesions in patients with a high risk of CRC. Furthermore, Kim et al. ${ }^{36}$ and Pickhardt et al. ${ }^{21}$ reported that CTC is comparable to OC in terms of the detection of clinically relevant lesions. They recommended CTC as the preferred screening strategy because it seems to achieve the 
same goals as colonoscopy (prevention and detection) but uses fewer resources and has a lower rate of complications. Graser et al. ${ }^{17}$ showed that high-resolution, low-dose CTC was comparable to OC in terms of sensitivity and may be considered an alternative screening test to colonoscopy. However, they also compared CTC and OC with sigmoidoscopy and FOBT. They concluded that for patients who refused full bowel preparation or either CTC or OC, sigmoidoscopy should be preferred over stool tests and fecal immunochemical tests should be preferred over FOBT. Therefore, on the basis of data reported in the literature, CTC and OC have a similar diagnostic accuracy and have no important differences in the detection of colorectal lesions in symptomatic high-risk patients. However, it must be emphasized that the performance of CTC is highly dependent on the size of the lesions and CTC has shown poor performance in detecting individual lesions $<5$ $\mathrm{mm}$, although the prevalence of malignancy in diminutive polyps is extremely small (approximately $0.25 \%$ ) and the probability of degeneration is very low. ${ }^{22}$ Ginnerup et al. ${ }^{23}$ reported that CTC and OC have similar overall detection rates for colorectal lesions $>6 \mathrm{~mm}$. Devir et al. ${ }^{24}$ and Gluecker et al. ${ }^{25}$ showed that CTC has good sensitivity and specificity for the detection of colorectal lesions $>10 \mathrm{~mm}$, although its accuracy in detecting polyps decreased as the size of the lesions decreased. These results are similar to those reported by Pineau et al., ${ }^{22}$ Laghi et al., ${ }^{15}$ and Hoppe et al. ${ }^{26}$ Additionally, Chaparro Sánchez et al. ${ }^{27}$ found that the sensitivity of CTC for the detection of colorectal lesions was highly variable and dependent on the lesion size: sensitivity was greater for larger lesions and considerably lower for lesions $<5 \mathrm{~mm}$. Flat lesions are difficult to detect with CTC because the conspicuity of flat lesions on three-dimensional endoluminal imaging is diminished, frequently resulting in false-negative findings. ${ }^{14}$ Flat polyps are more likely to be missed than sessile or pedunculated polyps, as flat polyps do not or only slightly alter the colonic contour. In several studies, flat lesions detected with conventional colonoscopy were missed with CTC; thus, flat lesions remain a diagnostic challenge for CTC. ${ }^{15,25}$ However, the detection of these lesions is important because $15-30 \%$ of CRCs originate from serrated polyps rather than from adenomatous polyps; therefore, preventive strategies must target not only adenomas but also all premalignant conditions. ${ }^{38}$ Ijspeert et al. ${ }^{39}$ conducted the first study that compared the detection rate of high-risk sessile serrated polyps (SSPs) between CTC and OC. They showed that flat SSPs with dysplasia located in the proximal colon were often not detected with CTC, probably because of the tendency of SSPs to flatten during the distension of the colon, called the "disappearing phenomenon", especially if they are flat and located in the cecum or in the ascending colon.
However, not all literature data confirm the similarity of CTC and $\mathrm{OC}$ in terms of diagnostic accuracy, sensitivity, and specificity for the detection of colorectal lesions, and there are controversial results across different studies. Johnson et al. ${ }^{28}$ reported a lower sensitivity of CTC in the detection of colorectal lesions than that reported in previous studies, which was probably because previous studies were based on cohorts with a high lesion prevalence. In addition, Spinzi et al. ${ }^{29}$ reported that CTC had poor sensitivity in detecting colorectal polyps and did not always detect neoplastic lesions or flat lesions. Cotton et al. ${ }^{30}$ concluded that the diagnostic accuracy of CTC is significantly inferior to that of OC and that CTC needs to be improved for widespread clinical application. However, data reported in the literature might have been influenced by various factors, and the discrepancies among various studies may be attributable to differences in bowel preparation, endoscopist and radiologist experience, scanning techniques, endoscopic measurement of lesions, and segmental localization. In terms of the preference of patients for CTC or OC, the literature reports heterogeneous data. Most patients may prefer CTC for CRC screening for reasons such as greater tolerability, less invasiveness, less fear of the procedure, reduced lower air insufflation, and lower risk of complications. ${ }^{40}$ Nevertheless, the use of premedication in $\mathrm{OC}$ has drastically changed the opinion of patients because it allows them to avoid experiencing pain, discomfort, and embarrassment by sleeping through the procedure. ${ }^{41}$

\section{CONCLUSIONS}

Colonoscopy is widely accepted as the gold standard for the detection of colorectal polyps and CRC and has the advantage of being both diagnostic and therapeutic; however, CTC is a better modality than OC for the visualization of the whole colon, detection of synchronous lesions, and accurate localization of tumors. The diagnostic accuracy of CTC has increased over time owing to improvements in scanning techniques, making it a valuable clinical screening method for colorectal neoplasms. It is widely accepted that the accuracy of CTC is comparable to that of colonoscopy for polyps $>10 \mathrm{~mm}$, acceptable but not equal to that of colonoscopy for lesions between 5 and $9 \mathrm{~mm}$, and poor for lesions $<5 \mathrm{~mm}$.

Conflicts of Interest

The authors have no potential conflicts of interest.

Funding

None. 
Author Contributions

Conceptualization: Alessia Chini, Michele Manigrasso

Data curation: Marco Milone

Formal analysis: Francesco Maione

Investigation: Grazia Cantore

Methodology: MMi

Project administration: AC

Resources: Rosa Maione

Supervision: Giovanni Domenico De Palma

Validation: AC, FM, GDDP

Visualization: FM

Writing-original draft: $\mathrm{MMa}$

Writing-review \& editing: AC

\section{ORCID}

\section{Alessia Chini}

Michele Manigrasso

Grazia Cantore

Rosa Maione

Marco Milone

Francesco Maione

Giovanni Domenico De Palma https://orcid.org/0000-0002-1851-1007 https://orcid.org/0000-0001-8204-7942 https://orcid.org/0000-0002-8984-0692 https://orcid.org/0000-0003-2672-9070 https://orcid.org/0000-0001-5612-1926 https://orcid.org/0000-0001-7043-9295 https://orcid.org/0000-0001-7474-7180

\section{REFERENCES}

1. Brenner H, Kloor M, Pox CP. Colorectal cancer. Lancet 2014;383:14901502.

2. Arnold M, Sierra MS, Laversanne M, Soerjomataram I, Jemal A, Bray F. Global patterns and trends in colorectal cancer incidence and mortality. Gut 2017;66:683-691.

3. Wong MC, Ding H, Wang J, Chan PS, Huang J. Prevalence and risk factors of colorectal cancer in Asia. Intest Res 2019;17:317-329.

4. Benson M, Dureja P, Gopal D, Reichelderfer M, Pfau PR. A comparison of optical colonoscopy and CT colonography screening strategies in the detection and recovery of subcentimeter adenomas. Am J Gastroenterol 2010;105:2578-2585.

5. De Rosa M, Pace U, Rega D, et al. Genetics, diagnosis and management of colorectal cancer (review). Oncol Rep 2015;34:1087-1096.

6. De Palma GD, Rega M, Masone S, et al. Conventional colonoscopy and magnified chromoendoscopy for the endoscopic histological prediction of diminutive colorectal polyps: a single operator study. World J Gastroenterol 2006;12:2402-2405.

7. de Wijkerslooth TR, de Haan MC, Stoop EM, et al. Study protocol: population screening for colorectal cancer by colonoscopy or CT colonography: a randomized controlled trial. BMC Gastroenterol 2010;10:47.

8. Mainenti PP, Salvatore B, D’Antonio D, et al. PET/CT colonography in patients with colorectal polyps: a feasibility study. Eur J Nucl Med Mol Imaging 2007;34:1594-1603.

9. Maggialetti N, Capasso R, Pinto D, et al. Diagnostic value of computed tomography colonography (CTC) after incomplete optical colonoscopy. Int J Surg 2016;33 (Suppl 1):S36-S44.

10. Moher D, Shamseer L, Clarke M, et al. Preferred reporting items for systematic review and meta-analysis protocols (PRISMA-P) 2015 statement. Syst Rev 2015;4:1.

11. Simon K. Colorectal cancer development and advances in screening. Clin Interv Aging 2016;11:967-976.

12. Morrin MM, Farrell RJ, Kruskal JB, Reynolds K, McGee JB, Raptopoulos V. Utility of intravenously administered contrast material at CT colonography. Radiology 2000;217:765-771.

13. Munikrishnan V, Gillams AR, Lees WR, Vaizey CJ, Boulos PB. Prospective study comparing multislice CT colonography with colonoscopy

in the detection of colorectal cancer and polyps. Dis Colon Rectum 2003;46:1384-1390.

14. Singh K, Narula AK, Thukral CL, Singh NR, Singh A, Kaur H. Role of CT colonography in colonic lesions and its correlation with conventional colonoscopic findings. J Clin Diagn Res 2015;9:TC14-TC18.

15. Laghi A, Iannaccone R, Carbone I, et al. Computed tomographic colonography (virtual colonoscopy): blinded prospective comparison with conventional colonoscopy for the detection of colorectal neoplasia. Endoscopy 2002;34:441-446.

16. Macari M, Bini EJ, Xue X, et al. Colorectal neoplasms: prospective comparison of thin-section low-dose multi-detector row CT colonography and conventional colonoscopy for detection. Radiology 2002;224:383392.

17. Graser A, Stieber P, Nagel D, et al. Comparison of CT colonography, colonoscopy, sigmoidoscopy and faecal occult blood tests for the detection of advanced adenoma in an average risk population. Gut 2009;58:241-248.

18. Iannaccone R, Laghi A, Catalano C, et al. Detection of colorectal lesions: lower-dose multi-detector row helical CT colonography compared with conventional colonoscopy. Radiology 2003;229:775-781.

19. Fenlon HM, Nunes DP, Schroy PC, Barish MA, Clarke PD, Ferrucci JT. A comparison of virtual and conventional colonoscopy for the detection of colorectal polyps. N Engl J Med 1999;341:1496-1503.

20. Yee J, Akerkar GA, Hung RK, Steinauer-Gebauer AM, Wall SD, McQuaid KR. Colorectal neoplasia: performance characteristics of CT colonography for detection in 300 patients. Radiology 2001;219:685692.

21. Pickhardt PJ, Choi JR, Hwang I, et al. Computed tomographic virtual colonoscopy to screen for colorectal neoplasia in asymptomatic adults. N Engl J Med 2003;349:2191-2200.

22. Pineau BC, Paskett ED, Chen GJ, et al. Virtual colonoscopy using oral contrast compared with colonoscopy for the detection of patients with colorectal polyps. Gastroenterology 2003;125:304-310.

23. Ginnerup Pedersen B, Christiansen TEM, Bjerregaard NC, Ljungmann $\mathrm{K}$, Laurberg S. Colonoscopy and multidetector-array computed-tomographic colonography: detection rates and feasibility. Endoscopy 2003;35:736-742.

24. Devir C, Kebapci M, Temel T, Ozakyol A. Comparison of 64-detector CT colonography and conventional colonoscopy in the detection of colorectal lesions. Iran J Radiol 2016;13:e19518.

25. Gluecker T, Dorta G, Keller W, Jornod P, Meuli R, Schnyder P. Performance of multidetector computed tomography colonography compared with conventional colonoscopy. Gut 2002;51:207-211.

26. Hoppe H, Netzer P, Spreng A, Quattropani C, Mattich J, Dinkel HP. Prospective comparison of contrast enhanced CT colonography and conventional colonoscopy for detection of colorectal neoplasms in a single institutional study using second-look colonoscopy with discrepant results. Am J Gastroenterol 2004;99:1924-1935.

27. Chaparro Sánchez M, del Campo Val L, Maté Jiménez J, et al. Computed tomography colonography compared with conventional colonoscopy for the detection of colorectal polyps. Gastroenterol Hepatol 2007;30:375380 .

28. Johnson CD, Harmsen WS, Wilson LA, et al. Prospective blinded evaluation of computed tomographic colonography for screen detection of colorectal polyps. Gastroenterology 2003;125:311-319.

29. Spinzi G, Belloni G, Martegani A, Sangiovanni A, Del Favero C, Minoli G. Computed tomographic colonography and conventional colonoscopy for colon diseases: a prospective, blinded study. Am J Gastroenterol 2001;96:394-400.

30. Cotton PB, Durkalski VL, Pineau BC, et al. Computed tomographic colonography (virtual colonoscopy): a multicenter comparison with standard colonoscopy for detection of colorectal neoplasia. JAMA 2004;291:1713-1719.

31. Mainenti PP, Romano M, Imbriaco M, et al. Added value of CT colo- 
nography after a positive conventional colonoscopy: impact on treatment strategy. Abdom Imaging 2005;30:42-47.

32. De Palma GD, Giglio MC, Bruzzese D, et al. Cap cuff-assisted colonoscopy versus standard colonoscopy for adenoma detection: a randomized back-to-back study. Gastrointest Endosc 2018;87:232-240.

33. Spada C, Stoker J, Alarcon O, et al. Clinical indications for computed tomographic colonography: European Society of Gastrointestinal Endoscopy (ESGE) and European Society of Gastrointestinal and Abdominal Radiology (ESGAR) guideline. Eur Radiol 2015;25:331-345.

34. Offermans T, Vogelaar FJ, Aquarius M, Janssen-Heijnen MLG, Simons PCG. Preoperative segmental localization of colorectal carcinoma: CT colonography vs. optical colonoscopy. Eur J Surg Oncol 2017;43:21052111.

35. Flor N, Ceretti AP, Luigiano C, et al. Performance of CT colonography in diagnosis of synchronous colonic lesions in patients with occlusive colorectal cancer. AJR Am J Roentgenol 2020;214:348-354.

36. Kim DH, Pickhardt PJ, Taylor AJ, et al. CT colonography versus colonoscopy for the detection of advanced neoplasia. N Engl J Med
2007;357:1403-1412.

37. Van Gelder RE, Nio CY, Florie J, et al. Computed tomographic colonography compared with colonoscopy in patients at increased risk for colorectal cancer. Gastroenterology 2004;127:41-48.

38. White TJ, Avery GR, Kennan N, Syed AM, Hartley JE, Monson JRT. Virtual colonoscopy vs conventional colonoscopy in patients at high risk of colorectal cancer--a prospective trial of 150 patients. Colorectal Dis 2009;11:138-145.

39. IJspeert JEG, Tutein Nolthenius CJ, Kuipers EJ, et al. CT-colonography vs. colonoscopy for detection of high-risk sessile serrated polyps. Am J Gastroenterol 2016;111:516-522.

40. Taylor SA, von Wagner C. Is CT colonography better tolerated than flexible sigmoidoscopy for colorectal cancer screening? Radiology 2018;286:884-886.

41. Jung HS, Park DK, Kim MJ, et al. A comparison of patient acceptance and preferences between CT colonography and conventional colonoscopy in colorectal cancer screening. Korean J Intern Med 2009;24:43-47. 